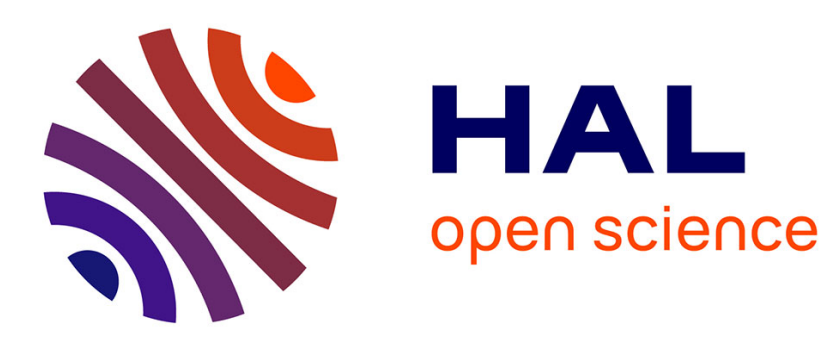

\title{
Distribution exacte du score local, cas Markovien
}

Sabine Mercier, Claudie Chabriac

\section{To cite this version:}

Sabine Mercier, Claudie Chabriac. Distribution exacte du score local, cas Markovien. Comptes rendus de l'Académie des sciences. Série I, Mathématique, 2003, 336 (10), pp.863-868. hal-00717186

\section{HAL Id: hal-00717186 \\ https://hal.science/hal-00717186}

Submitted on 12 Jul 2012

HAL is a multi-disciplinary open access archive for the deposit and dissemination of scientific research documents, whether they are published or not. The documents may come from teaching and research institutions in France or abroad, or from public or private research centers.
L'archive ouverte pluridisciplinaire HAL, est destinée au dépôt et à la diffusion de documents scientifiques de niveau recherche, publiés ou non, émanant des établissements d'enseignement et de recherche français ou étrangers, des laboratoires publics ou privés. 


\title{
Distribution Exacte du Score Local, Cas Markovien
}

\author{
Sabine MERCIER, Claudie HASSENFORDER
}

Université de Toulouse II, Equipe GRIMM,

Dpt Math-Info, UFR SES,

31100 Toulouse cedex 9, France.

Tel.: $05-61-50-40-22$

Fax : 05-61-50-41-73

E-mail: mercier@univ-tlse2.fr, chabriac@univ-tlse2.fr

Résumé.

Soit $\mathbb{X}=\left(X_{k}\right)_{k \geq 1}$ une suite de variables à valeurs dans $\{-v, \ldots, 0, \ldots,+u\}$. On définit le score local d'une séquence par $H_{n}=\max _{1 \leq i \leq j \leq n}\left(\sum_{k=i}^{j} X_{k}\right)$. Le score local est utilisé notamment dans l'analyse des séquences biologiques afin de mettre en évidence des régions de séquences ayant des propriétés biologiques intéressantes. La signification statistique des scores locaux calculés permet alors de mettre en évidence ce qui est réellement intéressant et il est donc nécessaire de connaître la distribution du score local. Nous établissons ici la loi exacte du score local dans le cas où la suite des $X_{i}$ est une chaîne de Markov d'ordre 1. 


\section{Exact Distribution for the Local Score of a Markov Chain.}

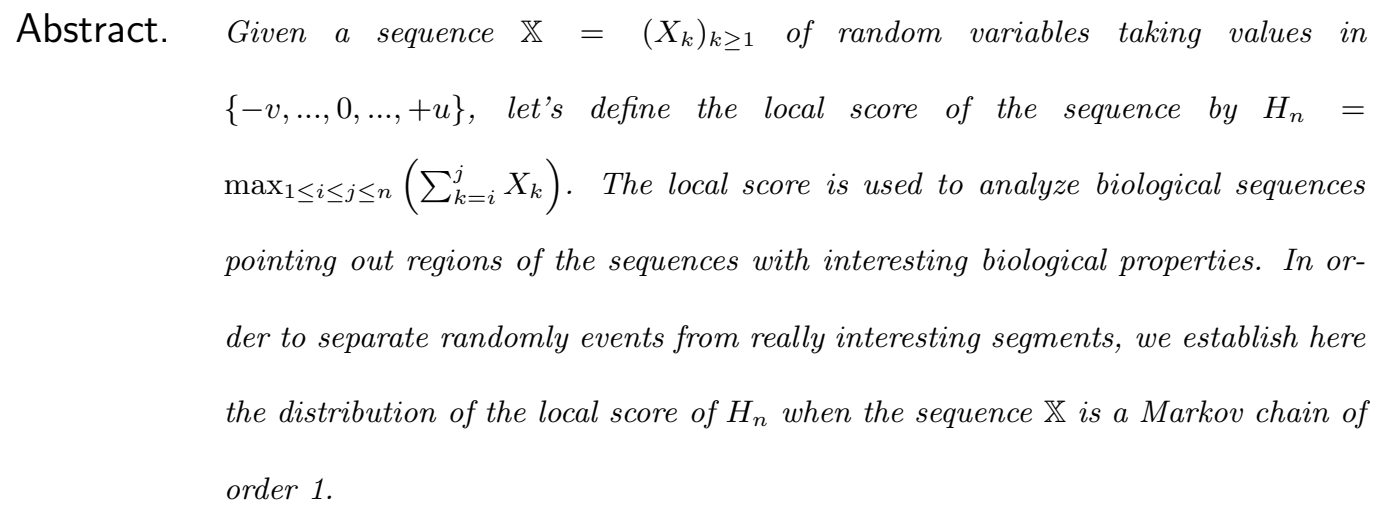

\section{Abridged English Version}

\section{Introduction}

Human Genome Project started in 1990, focusing on developing tools used to analyze the numerous biological sequences filling data banks. In order to point out segments with interesting biological properties, each component of the sequence is assigned a numerical value representing physicochemical properties as hydrophobicity, antigeniticity, ... These values are called scales or scores and are often used to analyze proteins. Let $s(k)$ be the score of the $k$-th component of a given sequence of length $n$. The local score is then defined by $H_{n}=\max _{1 \leq i \leq j \leq n} \sum_{k=i}^{j} s(k)$. Let's precise that the length of the segment which realizes the local score is not a priori known. The statistical problem is to establish the distribution of the local score to separate random events from really interesting segments. The distribution of $H_{n}$ was already established when the sequence of the scores $\mathbb{X}: X_{1}, \ldots, X_{n}$ is modelized as a sequence of independent and identically distributed random variables taking values in $\{-v, \ldots, 0, \ldots,+u\}$. See [6], [10] for asymptotic formula of the distribution when the average score is non positive, and [11] for the exact distribution in any average score. Markov chain is a more adequate model for biological sequence analysis and we give 
here the distribution of $H_{n}$ when $\mathbb{X}$ is a 1-order Markov chain.

\section{Results}

Let $\left(X_{k}\right)_{k \geq 1}$ be a 1-order Markov chain of probability matrix $\Lambda=\left(\Lambda_{i j}\right)_{i, j \in \mathbb{Z}}$ and $\gamma$ the initial distribution. The Markov chains will be implicitly of order 1 .

Let $P=\left(P_{(i, j)(k, \ell)}\right)$ be a matrix such that $(i, j)$ and $(k, \ell)$ belong to

$$
E=\{0, \ldots, a\} \times\{-v, \ldots, 0, \ldots,+u\} \quad \text { with } a \in \mathbb{N},
$$

and defined by

$$
\left\{\begin{array}{ll}
P_{(i, j)(0, \ell)}=\Lambda_{j \ell} \quad & \text { if } i+j \leq 0 \\
P_{(i, j)(i+j, \ell)}=\Lambda_{j \ell} & \text { if } 0<i+j \leq a-1 \\
P_{(i, j)(a, \ell)}=\Lambda_{j \ell} & \text { if } i+j \geq a \\
P_{(i, j)(k, \ell)}=0 & \text { else }
\end{array} .\right.
$$

Theorem 1 The statistical significance of the local score $H_{n}$ is given by

$$
(\forall a \geq 0) \quad P\left[H_{n} \geq a\right]=\sum_{j, \ell} \gamma_{j} \cdot P_{(0, j)(a, \ell)}^{n}
$$

Let $S_{k}$ be the partial sums of the sequence $\mathbb{X}: S_{0}=0$ and $S_{k}=X_{1}+\ldots+X_{k}$. Let $T_{k}$ be the following stopping times : $T_{0}=0$ and $T_{k+1}=\inf \left\{i>T_{k} ; S_{i}-S_{T_{k}}<0\right\}$. Consider the process $U$ defined by : $U_{0}=0$ and for $T_{k} \leq j<T_{k+1}, U_{j}=S_{j}-S_{T_{k}}$. We have

Lemma $1 U_{j}=\max \left(U_{j-1}+X_{j}, 0\right)=\left(U_{j-1}+X_{j}\right)^{+} \quad$ and $\quad H_{n}=\max _{1 \leq k \leq n} U_{k}$

Let $U^{*}$ be the process stopped in $a$, with $a \in \mathbb{N}^{*}$. We get $U_{j}^{*}=U_{j}$ if $j<\tau_{a}$ and $U_{j}^{*}=a$ if $j \geq \tau_{a}$ with $\tau_{a}=\inf \left\{j \geq 1 ; U_{j} \geq a\right\}$. And finally, let's define the sequence $\mathbb{Y}$ by $: Y_{n+1}=\left(U_{n}^{*}, X_{n+1}\right)$ for $n \geq 0$

Lemma $2 \mathbb{Y}$ is a Markov chain with probability matrix $P=\left(P_{(i, j)(k, \ell)}\right)_{(i, j)(k, \ell) \in E}$, and $P_{(i, j)(k, \ell)}=$ $P\left[\left(U_{n}^{*}=k\right) \cap\left(X_{n+1}=\ell\right) \mid\left(U_{n-1}^{*}=i\right) \cap\left(X_{n}=j\right)\right]$, determined in (2). 
Lemma 3 The distribution of $U^{*}$ is given by

$$
P\left[U_{n}^{*}=k\right]=\sum_{j, \ell} \gamma_{j} \cdot P_{(0, j)(k, \ell)}^{n}
$$

From Lemma 1, we deduce $P\left[H_{n} \geq a\right]=P\left[U_{n}^{*}=a\right]$ and using Lemma 3 and the explicitation of the $P_{(i, j)(k, \ell)}$, Theorem 1 is proved. The determination of the $P_{(i, j)(k, \ell)}$ is easy, using elementary notions of Markov Chain Theory.

\section{Conclusion and perspectives}

Using both the exact distribution of $H_{n}$ in i.i.d. and markovian case, we are going to compare these two models with an empirical distribution calculated on real biological sequences. This comparison will stand on exact formulas and thus will focus on the models only.

\section{Version française}

\section{Introduction}

La biostatistique est une discipline en pleine expansion de même que toutes les disciplines en rapport avec le génome et les bases de données de séquences biologiques croissent de façon exponentielle. Nous sommes maintenant dans l'ère de l'après séquençage où l'information doit être extraite de ces nouvelles données. Un des objectifs du projet Génome Humain, débuté en 1990, consiste à développer et à améliorer les outils d'analyse de séquences. Il existe actuellement de nombreux outils permettant d'extraire de l'information de ces séquences. Plusieurs portent sur l'analyse de la structure primaire, c'est-à-dire sur la connaissance de la succession des bases azotées (ou nucléotides) ou des acides aminés (pour les protéines), et déterminent des profils de protéines ou d'ADN à partir d'échelles. Une échelle d'acides aminés associe à chaque type d'acides aminés une 
valeur numérique également appelée score et déterminée expérimentalement ou statistiquement. Les échelles d'acides aminés les plus fréquemment utilisées sont les échelles d'hydrophobicité et celles correspondant aux paramètres de conformation de structure secondaire. Ces profils mettent en évidence des parties ou segments de séquences les plus pondérées suivant l'échelle utilisée. Soit $H_{n}$ le score local correspondant au score maximal observé, en considérant tous les segments de la séquence de longueur $n$, à n'importe quelle position, de toutes les longueurs possibles. Nous avons

$$
H_{n}=\max _{1 \leq i \leq j \leq n} \sum_{k=i}^{j} s(k),
$$

avec $s(k)$ le score du kème élément de la séquence.

Afin de distinguer l'information pertinente du simple hasard, il est important d'établir la signification statistique du score local. De nombreux travaux portent sur l'établissement de la loi de $H_{n}$, qui nécessite avant tout de définir un modèle sur les séquences biologiques, ou bien sur les séquences des scores correspondantes.

Soit donc $X_{1} \ldots X_{n}$ une suite de variables aléatoires à valeurs dans $\{-v, \ldots, 0, \ldots,+u\},-v$ et $+u$ étant respectivement les minimum et maximum de l'échelle choisie pour l'étude. Le cas où les variables $X_{k}$ sont indépendantes et identiquement distribuées a fait l'objet de nombreux travaux. Voir par exemple [12], [4] pour une revue des problèmes et résultats sur le sujet. La distribution exacte du score local a été établie dans le cas i.i.d. dans [2] et [11]. Ce résultat a trois avantages par rapport aux précédents travaux portant sur la loi du score local. Le premier est dû au caractère exact du résultat, contrairement aux approximations utilisées jusque là, [5], [6]. Le deuxième intérêt, repose sur le fait que le score moyen $E\left(X_{k}\right)$ n'a pas besoin d'être négatif. En effet, les travaux de Karlin et al. [5], [6], de Mercier et al. [10] [9], ainsi que ceux de Waterman et al. se placent dans le cas où l'espérance du score est négative (on a alors $H_{n}=\mathcal{O}(\ln n)$ ), mais ne sont pas valables si cette espérance est positive (dans ce cas $H_{n}=\mathcal{O}(n)$ ). Le résultat de Daudin et al. [2], [11] est quant à 
lui valable indépendamment du signe du score moyen. Enfin, un troisième aspect particulièrement intéressant concerne la longueur des séquences étudiées. En effet, les approximations de la loi du score local sont asymptotiques par rapport à la longueur des séquences. Or, les protéines ont une longueur moyenne de 350 acides aminés et ne rentrent pas dans ce cadre asymptotique. Certes, le caractère exact de [11] résout ce problème, mais l'implémentation du résultat sera d'autant plus précise et rapide à obtenir (cumul d'arrondis de calculs) que les séquences seront courtes.

Le but de cette note consiste à établir la loi de $H_{n}$ dans le cas où les séquences sont modélisées par une chaîne de Markov d'ordre 1. Nous travaillons directement sur la suite des scores c'est-à-dire sur des suites à valeurs dans $\mathbb{Z}$. La loi du score local dans le cas markovien est énoncée dans le

Théorème 1. Les démonstrations utilisent des notions élémentaires de la théorie des chaînes de Markov.

Toutes les chaînes de Markov sont d'ordre 1, même si cela n'est pas précisé chaque fois.

\section{Résultats et démonstations}

Soit $\left(X_{k}\right)_{k \geq 1}$ une chaîne de Markov d'ordre 1, de matrice de transition $\Lambda=\left(\Lambda_{i j}\right)_{i, j \in \mathbb{Z}}$ et de loi initiale $\gamma$. Le score local de la suite $\left(X_{k}\right)$ est défini par $H_{n}=\max _{1 \leq i \leq j \leq n}\left(\sum_{k=i}^{j} X_{k}\right)$. Soit

$$
E=\{0, \ldots, a\} \times\{-v, \ldots, 0, \ldots,+u\} \quad \text { avec } a \in \mathbb{N}
$$

Introduisons la matrice $P=\left(P_{(i, j),(k, \ell)}\right)$, où $(i, j)$ et $(k, \ell)$ sont dans $E$, défini par

$$
\begin{cases}P_{(i, j)(0, \ell)}=\Lambda_{j \ell} & \text { si } i+j \leq 0 \\ P_{(i, j)(i+j, \ell)}=\Lambda_{j \ell} & \text { si } 0<i+j \leq a-1 \\ P_{(i, j)(a, \ell)}=\Lambda_{j \ell} & \text { si } i+j \geq a \\ P_{(i, j)(k, \ell)}=0 & \text { sinon }\end{cases}
$$

Nous avons le résultat suivant 
Théorème 1 La signification statistique du score local $H_{n}$ est donnée par la formule suivante

$$
(\forall a \geq 0) \quad P\left[H_{n} \geq a\right]=\sum_{-v \leq j, \ell \leq+u} \gamma_{j} \cdot P_{(0, j)(a, \ell)}^{n} .
$$

\section{Démonstration}

Notons $S_{k}$ les sommes partielles associées à la suite des $X_{k}: S_{0}=0$ et $S_{k}=X_{1}+\ldots+X_{k}$. Considérons la suite des temps d'arrêt $T_{k}$ définie par : $T_{0}=0$ et $T_{k+1}=\inf \left\{i>T_{k} ; S_{i}-S_{T_{k}}<0\right\}$. Par définition des $T_{k}$, la suite des $S_{T_{k}}$ est strictement décroissante, et les $T_{k}$ sont appelés les temps successifs des records négatifs.

Soit $U$ la suite définie de manière récurrente par : $U_{0}=0$ et pour $T_{k} \leq j<T_{k+1}, U_{j}=S_{j}-S_{T_{k}}=$ $X_{T_{k}+1}+\ldots+X_{j}$. On a en particulier $U_{T_{k}}=0$ pour tout $k \geq 0$. Les $\left(U_{j}\right)$ forment une suite positive non nécessairement bornée. Comme démontré dans [2] et [11], nous avons les résultats suivants :

\section{Lemme 1}

$$
U_{j}=\max \left(U_{j-1}+X_{j}, 0\right)=\left(U_{j-1}+X_{j}\right)^{+} \quad \text { et } \quad H_{n}=\max _{1 \leq k \leq n} U_{k}
$$

Notons $U^{*}$ le processus arrêté de $U$ en $a$, où $a$ est dans $\mathbb{N}^{*}$.

$$
U_{j}^{*}=U_{j} \quad \text { si } j<\tau_{a} \quad \text { et } \quad U_{j}^{*}=a \quad \text { si } j \geq \tau_{a} \quad \text { avec } \quad \tau_{a}=\inf \left\{j \geq 1 ; U_{j} \geq a\right\}
$$

Le processus $\left(U_{j}^{*}\right)$ est une chaîne de Markov non homogène à valeurs dans $\{0,1, \ldots, a\}$.

Dans le cas d'une suite $\left(X_{k}\right)$ i.i.d. (voir [2] et [11]), $U^{*}$ est une chaîne de Markov homogène et il est alors facile d'établir la loi de $U_{n}^{*}$, ce qui n'est plus vrai dans le cas d'une suite $\left(X_{k}\right)$ markovienne. Afin d'expliciter la loi de $U_{n}^{*}$, considérons la chaîne $\left(Y_{n}\right)$ définie par

$$
(\forall n \geq 0) \quad Y_{n+1}=\left(U_{n}^{*}, X_{n+1}\right)
$$

qui est homogène et dont l'ensemble des états est $E$ défini en (3). 
Lemme 2 (Matrice de transition de $Y)\left(Y_{n}\right)_{n \geq 1}$ est une chaîne de Markov de matrice de transition $P=\left(P_{(i, j)(k, \ell)}\right)$, avec $(i, j)$ et $(k, \ell)$ dans $E$, où les $P_{(i, j)(k, \ell)}$ sont donnés par $(4)$.

$$
P\left[\left(U_{n}^{*}=k\right) \cap\left(X_{n+1}=\ell\right) \mid\left(U_{n-1}^{*}=i\right) \cap\left(X_{n}=j\right)\right]=P_{(i, j)(k, \ell)} .
$$

Les $P_{(i, j)(k, \ell)}$ sont déterminés à l'aide de $\Lambda$ comme indiqué dans le Théorème 1.

\section{Lemme $3\left(\right.$ Loi de $\left.U_{n}^{*}\right)$}

$$
P\left[U_{n}^{*}=k\right]=\sum_{j, \ell} \gamma_{j} \cdot P_{(0, j)(k, \ell)}^{n}
$$

Du Lemme 1, on tire alors $P\left[H_{n} \geq a\right]=P\left[U_{n}^{*}=a\right]$. En utilisant ensuite le Lemme 3 et l'explicitation des $P_{(i, j)(k, \ell)}$, le Théorème 1 est alors démontré.

\section{Démonstration du Lemme 2}

Nous avons pour $i=a, P_{(a, j)(k, \ell)}=0$ si $k \leq a-1$ car $U^{*}$ est un processus arrêté en $a$, et $P_{(a, j)(k, \ell)}=$ $\Lambda_{j \ell}$ pour $k=a$. Pour $i \neq a$, alors : $P_{(i, j)(k, \ell)}=P\left[\left(U_{n}^{*}=k\right) \cap\left(X_{n+1}=\ell\right) \mid\left(U_{n-1}=i\right) \cap\left(X_{n}=j\right)\right]$.

- Si $k=0$, alors

$$
\begin{aligned}
P_{(i, j)(0, \ell)} & =P\left[\left(X_{n} \leq-U_{n-1}\right) \cap\left(X_{n+1}=\ell\right) \mid\left(U_{n-1}=i\right) \cap\left(X_{n}=j\right)\right] \\
& =P\left[(j \leq-i) \cap\left(X_{n+1}=\ell\right) \mid\left(U_{n-1}=i\right) \cap\left(X_{n}=j\right)\right] \\
& =P\left[(j \leq-i) \cap\left(X_{n+1}=\ell\right) \mid\left(X_{n}=j\right)\right]
\end{aligned}
$$

car $U_{n-1}$ ne dépend que de $X_{1}, \ldots, X_{n-1}$ et $X_{n}$ est une chaîne de Markov d'ordre 1 par hypothèse. D'où, $P_{(i, j)(0, \ell)}=\Lambda_{j \ell}$ si $j \leq-i$ et 0 sinon.

- Si $0<k \leq a-1$, alors

$$
P_{(i, j)(k, \ell)}=P\left[\left(U_{n-1}+X_{n}=k\right) \cap\left(X_{n+1}=\ell\right) \mid\left(U_{n-1}=i\right) \cap\left(X_{n}=j\right)\right]
$$




$$
\begin{aligned}
& =P\left[(i+j=k) \cap\left(X_{n+1}=\ell\right) \mid\left(U_{n-1}=i\right) \cap\left(X_{n}=j\right)\right] \\
& =\Lambda_{j \ell} \text { si } k=i+j \text { et } 0 \text { sinon }
\end{aligned}
$$

- Pour $k=a$, nous avons

$$
\begin{aligned}
P_{(i, j)(a, \ell)} & =P\left[\left(X_{n} \geq a-U_{n-1}\right) \cap\left(X_{n+1}=\ell\right) \mid\left(U_{n-1}=i\right) \cap\left(X_{n}=j\right)\right] \\
& =P\left[(j \geq a-i) \cap\left(X_{n+1}=\ell\right) \mid\left(U_{n-1}=i\right) \cap\left(X_{n}=j\right)\right] \\
& =\Lambda_{j \ell} \text { si } i+j \geq a \text { et } 0 \text { sinon } .
\end{aligned}
$$

\section{Perspectives}

Ayant à notre disposition la loi exacte du score local dans le cas où les séquences sont modélisées par une suite de variables aléatoires indépendantes et identiquement distribuées, cf. [2] et [11], ainsi que maintenant dans le cas markovien, nous avons ici la possibilité de comparer des modèles sans que les conclusions ne soient altérées par le fait que l'on aura utilisé des approximations.

\section{References}

[1] Arratia, R.and Waterman, M.-S. (1994). A phase transition for the score in matching random sequences allowing deletions. Ann. Appl. Prob., 4:200-225.

[2] Daudin, J.-J. And Mercier, S. (1999). Distribution exacte du score local d'une suite de variables indépendantes et identiquement distribuées. C. R. Acad. Sc. Paris, 329(1):815-820.

[3] Dembo, A., Karlin, S. (1991). Strong limit theorems of empirical functionnals for large exceedences of partial sums of i.i.d. variables. Ann. Prob., 19(4):1737-1755.

[4] Durbin, R., Eddy, S., Krogh, A., And Mitchison, G. (1998). Biological Sequence Analysis. Probabilistic Models of Proteins and Nucleic Acids. Cambridge University Press, Cambridge, U.K. 
[5] Karlin, S., and Altschul, S.F. (1990). Methods for assessing the statistical significance of molecular sequence features by using general scoring schemes. Proc. Natl. Acad. Sci. USA, 87, 2264-2268.

[6] Karlin, S., And Dembo, A. (1992). Limit distributions of maximal segmental score among Markov-dependent partial sums. Adv. Appl. Prob., 24, 113-140.

[7] Karlin, S., and Taylor, H.M. (1981). A second course in stochastic processes. Academic Press.

[8] Kyte, J., And Doolittle, R.F. (1982). A simple method for displaying the hydrophatic character of a protein. J. Mol. Biol., 157, 105-132.

[9] Mercier, S. (1999). Statistiques des scores pour l'analyse et la comparaison de séquences biologiques. Thèse de doctorat d'Université, Rouen.

[10] Mercier, S., Cellier, D., F. Charlot and Daudin, J.-J. (2001). Exact and asymptotic distribution for the local score of one i.i.d. random sequence. LNCS, volume for JOBIM 2000, (2066):74-85.

[11] Mercier, S.And Daudin, J.-J. (2001). Exact distribution for the local score of one i.i.d. random sequence. J. Comp. Biol., 8(4):373-380.

[12] Waterman, M.S. (1995). Introduction to Computational Biology. Chapman and Hall, London. 